\title{
Segmental definition: III. Definitive basis for distinguishing somatic findings of visceral reflex origin
}

\author{
WILLIAM L. JOHNSTON, DO, FAAO \\ East Lansing, Michigan
}

(Part I of this series showed how somatic dysfunction is examined by application of a series of passive gross motion tests. Part II gave an indirect method in osteopathic manipulative treatment.) Somatic motor components of visceral disease have been mentioned frequently in the literature, but seldom with sufficient descriptors to help physicians detect them on physical examination. Further, descriptors that would distinguish the motor effects of visceral inputs from other somatic findings have seldom been discussed. Criteria for recognizing linkage of vertebral and costal asymmetries at the same thoracic spinal level as a distinctive motor component of visceral reflex origin are summarized. Four clinical examples show predictable locations of specific segmental asymmetries that have been observed with increased frequency in patients with certain kinds of disease. During osteopathic manipulative management of well-established segmental dysfunction with linkage, the precise character of the observed segmental asymmetry has been shown to persist. Recognition of this feature poses the question in neuromuscular physiology of how specific segments become programmed with a precise asymmetric response to motion when inputs along visceral afferents are involved.

The motion tests for segmental definition of symmetry/asymmetry described in parts 1 and 2 of this series provide raw clinical data about motor function at the segments of a human mobile system. In this instance, the term raw data implies that a location of dysfunction has been identified, motion tests for securing the information have been described, and criteria for judging the presence of motion asymmetry have been established. The situation thus presents enough objective features to predict reproducibility by trained examiners.

In the past, because one or more of these features has been absent, clinicians often have been unable to reproduce, on physical examination, those somatic findings that have been reported as manifestations of visceral disease. Location sometimes has been the only item described; this provides no clues for distinguishing findings of somatic origin from those of visceral origin at the given location. Frequently, the testing procedure has not been described at all. As a result, the finding has been characterized conceptually, rather than factually within the context of the testing procedure. Especially when the criterion for a finding is subjective tenderness on pressure, the objectivity is seriously limited and reproducibility less reliable.

In previous work, our hypothesis has been that, when factually reported, the raw data concerning the individuality of segmental asymmetries and their arrangements within the mobile system would provide clues to distinguish between the somatic and visceral reflex components that are manifested as motor changes in somatic tissues. In describing the motion tests and establishing criteria for findings, we have tried to avoid those interpretations of vertebral mobility that have emerged primarily during vertebral positional diagnosis. An example is the finding of a vertebral transverse process that is more posterior on the right; posi- 


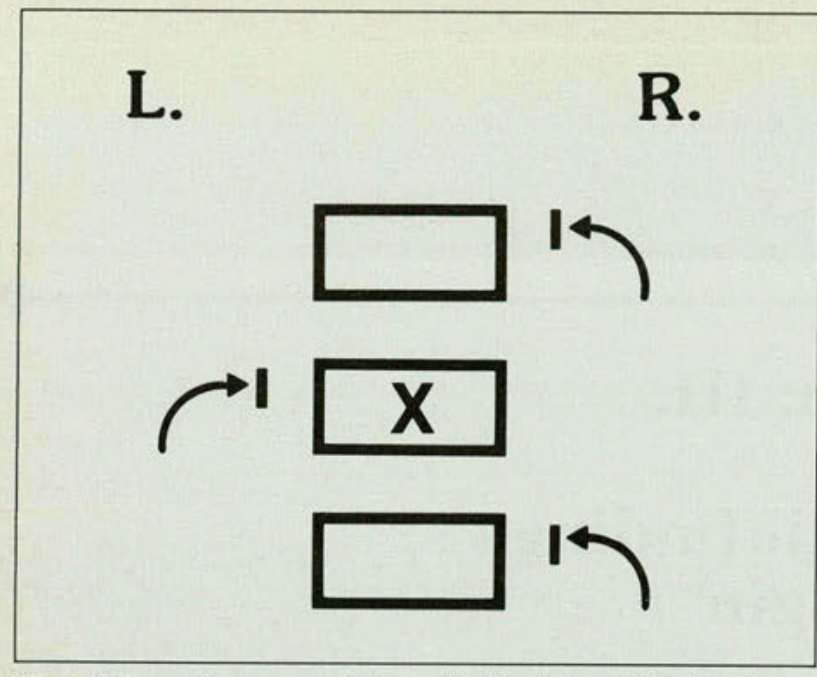

Fig 1.Schematic representation of a fundamental unit of segmental dysfunction: A primary motor asymmetry is indicated at central segment $\mathrm{X}$, resisting rotation right, with compensatory opposing asymmetries at adjacent segments resisting rotation left.

tional diagnosis of the spinous processes, separated or approximated (flexed or extended) on the vertebra below, implies sidebent right and restricted in sidebending left.

On the other hand, even the restriction found during motion tests has often been interpreted as muscle spasm, fascial tightness, facet lock, or ligamentous block. For example, once viewed within the concept of facet lock, restriction has often been considered to involve some directions of joint motion not actually tested. For these reasons, we temporarily set aside the positional aspects of segmental diagnosis and turned to a description of positive findings on motion tests that would provide the raw data about the individuality of segmental motion asymmetries and clues to the presence of motor components of visceral reflex origin.

\section{Somatic motor findings of somatic reflex origin}

Part 2 of this report considered the palpable evidence of a secondary somatic reflex response to a primary somatic motion defect. In Fig 1, asymmetry at the primary lesioned segment at $X$ is accompanied by secondary somatic asymmetries at adjacent segments (superior and inferior). Consideration of the motor effects of somatosomatic reflexes in the spine has not always included the idea of adaptation occurring above and below a primary defect.

A more familiar example is shown in Fig 2A, where the defect is present as a laterally inclined base of support with compensatory changes only appearing above, in the superimposed spinal re-

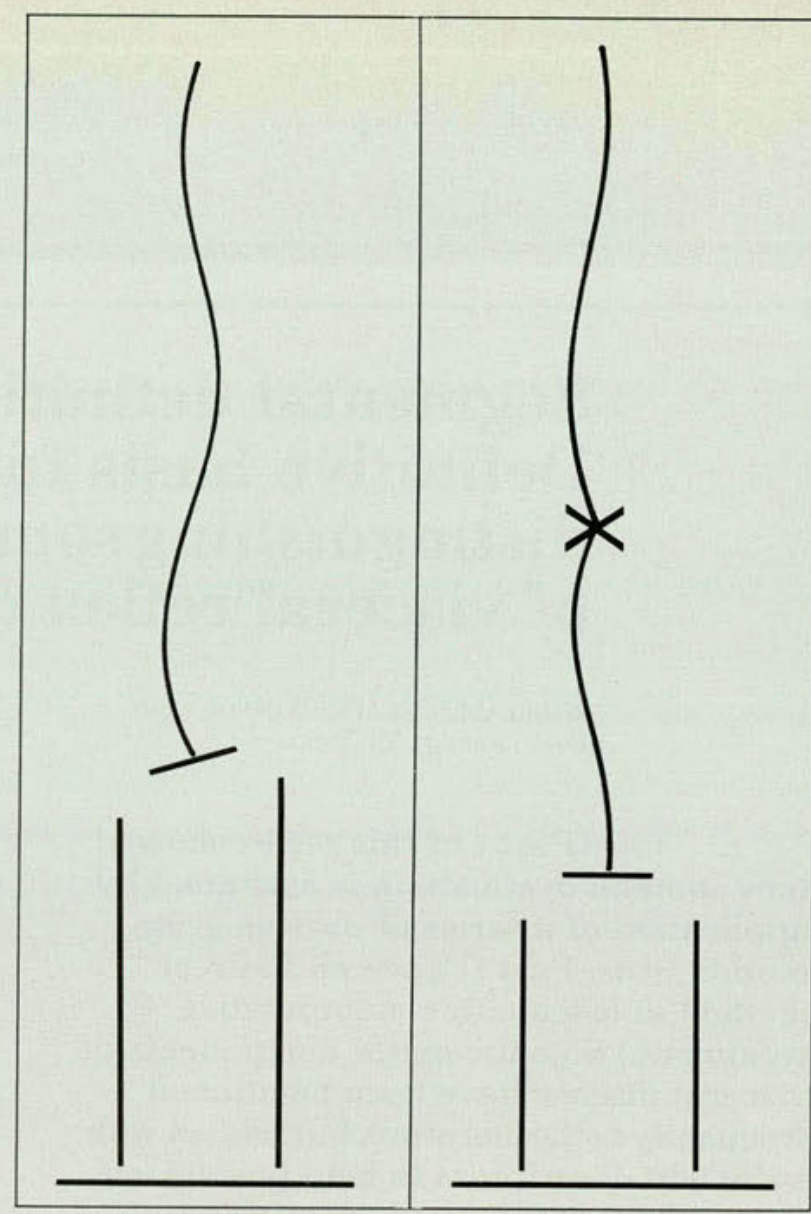

Fig 2A.Spinal adaptation to an asymmetric base of support inclined to the left, illustrating secondary scoliosis with spinal curves convexed in left lumbar and right thoracic areas.

Fig 2B.Spinal adaptation to a central primary segmental defect at $\mathrm{X}$, with secondary spinal curves convexed left, superiorly and inferiorly, with relative symmetry at the base of support.

alignment. It is not unusual, however, to recognize clinically the differing spinal configuration presented in Fig 2B. Examination should be made with the patient standing, with the spine in the forwardbent, semiflexed position to emphasize the presence of lateral spinal curvature. Although symmetry often exists at the base of support in the pelvis, these compensatory spinal deviations are found, convexed in the same direction (here to the left), above and below a primary midspinal defect indicated at $X$.

Primary defects of this kind often are associated with a history relating to childhood illness or injury. The somatic reflex response initiates compensatory spinal patterns above and below, and these may become relatively fixed in postural development during growth. Examination of the adult posture frequently provides such examples of the motor effects of somatosomatic reflex activity. 
Up to this point, these examples of somatosomatic reflexes have considered relationships in only the vertical column of spinal vertebral segments. However, in the thoracic region, the mobile system actually presents three vertical mobile columns - one central vertebral and two lateral costal columns. Each is accessible to examination using the same kinds of motion testing procedures that were described before (passive rotations and translations introduced through the shoulders and trunk, and active respiration). Fundamental threesegment units of dysfunction can present a solitary focus of dysfunction in the vertebral column or in one of the costal columns, quite separately. Somatosomatic reflections occur as mirror-image motion asymmetries at adjacent vertebral segments for a vertebral dysfunction, and at adjacent costal segments for a costal dysfunction, each in their vertical alignment.

When compensatory (secondary) changes occur horizontally, across adjacent columns, the principle of mirror-image asymmetries in somatosomatic reflex processes still applies. That is, in adjacent dysfunctional segments at the same spinal level, a comparison of vertebral and costal findings on motion tests will show opposing directions of motion asymmetries. An example is shown in Fig $3 \mathrm{~A}$, which schematically represents a primary dysfunction $(\mathrm{X})$ at left rib 7 resisting active inhalation, and passive gross axial rotation of shoulders and trunk to the left, as indicated. (Test findings also would include resistance to passive sidebending to the left, extension, and translations anteriorly, to the right, and caudally.)

Secondary mirror-image asymmetries are present at adjacent left ribs 6 and 8 . Repeating the passive gross motion tests, but palpating for response in the adjacent central column at vertebral segments $\mathrm{T} 6, \mathrm{~T} 7$, and $\mathrm{T} 8$, reveals an opposite set of asymmetries to the fundamental unit of dysfunction in the left lateral column at rib 7, as indicated by arrows in Fig. 3A (T7 resists rotation right and $\mathrm{T} 6$ and $\mathrm{T} 8$ resist rotation left). The adaptive nature of the vertebral column asymmetry again is revealed after successful resolution of the primary dysfunction at left rib 7 . This is achieved by applying an indirect method guided by the palpatory cues of rotary, translatory, and respiratory asymmetries at left rib 7 only. Re-examination demonstrates a return to symmetric response to motion testing at both central vertebral and left costal columns throughout the mobile complex of six segments.

Confidence in the predictability of this complex of palpatory findings and its resolution within the thoracic region has supported the phenomenon of mirror-image asymmetries at adjacent segments,

\section{VERTEBRAL}

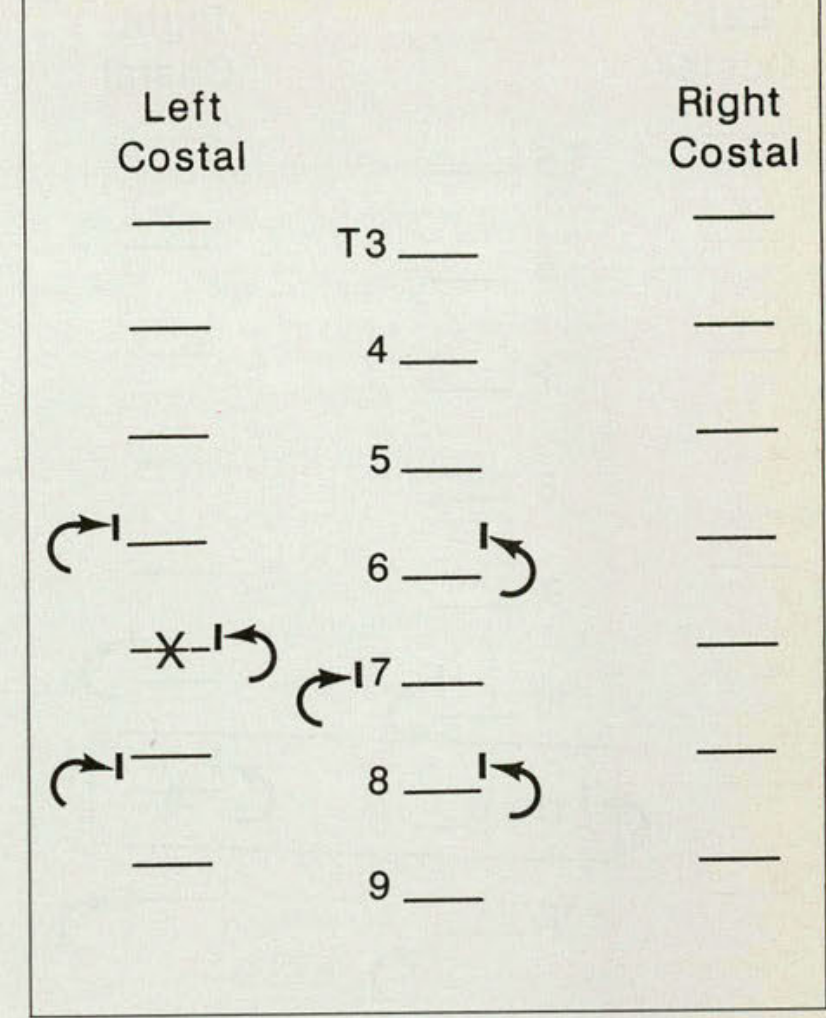

Fig 3A.Schematic representation of primary dysfunction $(X)$ at left rib 7 resisting active inhalation and passive rotation to the left. Secondary asymmetries are indicated at left ribs 6 and 8 within the left lateral vertical column. Opposing vertebral asymmetries at T-6, T-7, and T-8 indicate a horizontal adaptation to the lateral costal dysfunction at left rib 7. (Arrows indicate directions of resistance to axial rotation tests only.)

vertical or horizontal, as singularly characteristic of a somatic finding of somatic reflex origin. The vertical adaptation to a primary motion defect is observed clinically to be the rule, whereas horizontal adaptation has been observed less frequently.

\section{Somatic motor findings of visceral reflex origin}

Once the somatic reflex basis for this characteristic behavior between adjacent segments was recognized, my coworkers and I, in a previous study, looked for variations that might express a possible distinctive feature of somatic findings of visceral reflex origin. The feature recognized has been termed linkage, which describes the palpable phenomenon of motor dysfunction recorded during our investigation ${ }^{1}$ of patients with renal disease. Linkage exists when there are identical motion asym- 


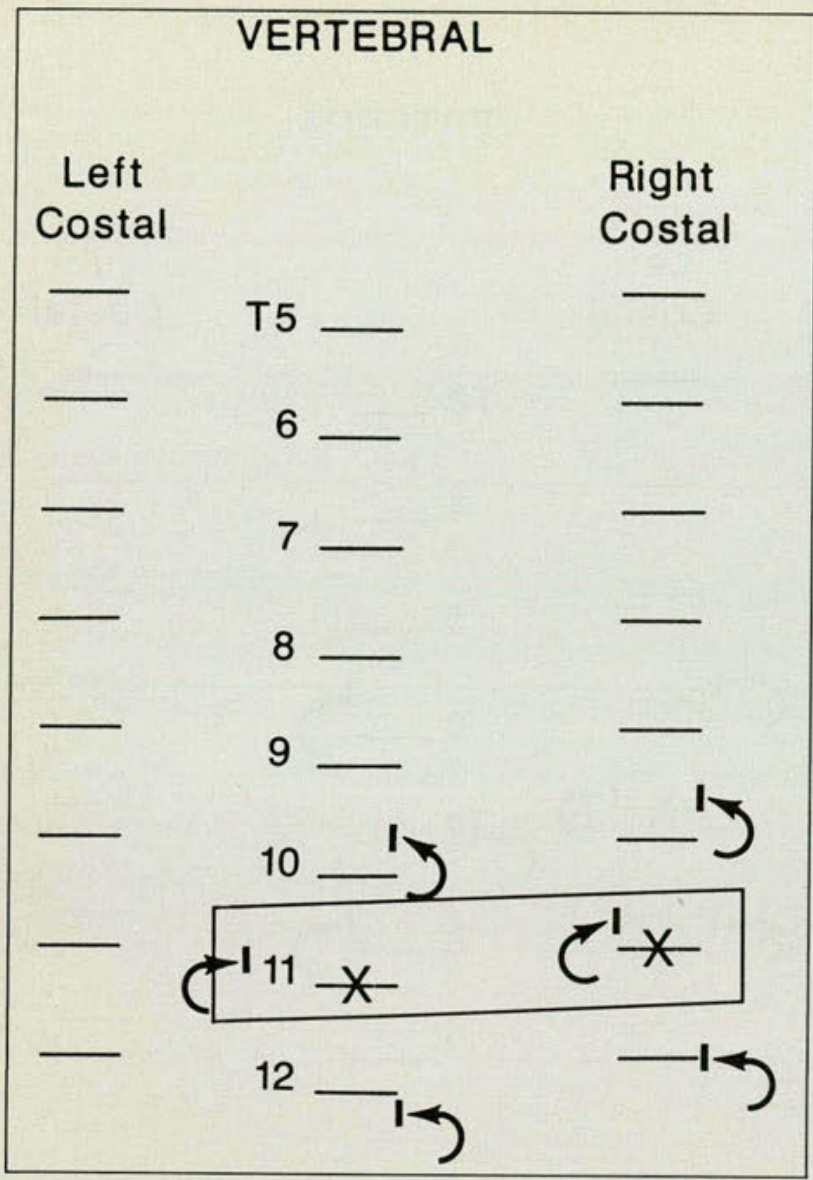

Fig 3B.Schematic representation of linkage at $T-11$ with right rib 11. Arrows at each indicate resistance to passive axial rotation to the right. Opposing asymmetries are indicated for adjacent segments (superiorly and inferiorly) in each vertical column.

metries at a vertebral and a costal dysfunction at the same thoracic segmental level; an example at T-11 level is shown in Fig 3B.

For this characteristic of linkage to be even considered as visceral in origin, its presence needed to satisfy certain clinical criteria. The motor component of reflex activity along specific visceral afferent pathways should be expected to register the following features: (1) similar segmental location in different patients with the same visceral problem (anatomic differences could account for variance in vertebral findings of one segment, above or below); and (2) similar identity in motion characteristics for a given segmental location with a specific visceral relationship. Certainly, when clinical observation reveals asymmetric spinal findings with similar motion characteristics at identical locations, occurring with increased frequency in the presence of a particular disease entity, a firm clinical relationship between the spinal and visceral findings must be considered.
The following descriptions include some predictable locations and motion characteristics of segmental asymmetries that have been observed with increased frequency in patients with the following disorders: (1) hypertension; (2) a history of bronchial asthma; (3) recurring acute upper gastrointestinal complaint (for example, repeated attacks of gastritis); and (4) cholelithiasis.

The first recognition of linkage came about in observation of hypertensive patients in private practice. My report ${ }^{2}$ of somatic patterning in the cervicothoracic region included description of characteristic findings of linkage at T-2 and T-6. (The pattern was described with a palpable asymmetry also at C-6, and this finding seemed to be consistently present in association with the findings at T-2 and T-6.)

At the T-2 level, linkage involves similar asymmetries in the central column and the left costal column. Findings at T2 and left rib 2 include immediately increasing local tissue resistance to the following: passive gross axial rotation introduced through the shoulders and trunk to the right, sidebending right, and extension; translations anteriorly, left, and caudally; and active inhalation. The opposing asymmetries (somatosomatic) are present at adjacent (superior and inferior) vertebral and left costal segments 1 and 3 , in compensation for the primary dysfunction at the T-2 level.

At T-6, linkage involves similar asymmetries in the central column and in the right costal column. Findings at T-6 and right rib 6 include increasing resistance to the following: passive axial rotation of shoulders to the left, sidebending right, and forward flexion; translations anteriorly, left, and caudally; and exhalation. The opposing asymmetries are present at adjacent vertebral and right costal segments 5 and 7 .

Results of interexaminer studies ${ }^{3,4}$ have indicated confidence in the reproducibility of pattern findings by trained examiners. Examiners made decisions on selected tests for findings of asymmetry in the vertebral column only, as a basis for the evaluation of presence or absence of the pattern in the patients examined. The motion tests selected for these studies were for response to anterior/ posterior, and lateral passive translations, and to active respiratory excursion. Vertebral levels tested (C-6, T-2, T-6) also included the adjacent segments (C-5 and C-7, T- 1 and T- 3 , and T- 5 and T7). 3,4 The studies confirmed an increased frequency in patients with moderate to severe hypertension as compared with those with normal blood pressure, in a ratio exceeding $2: 1$.

Clues to involvement at the T-4 level in patients with a history of bronchial asthma came originally 
from experiences with P.T. Wilson, DO, during internship at Massachusetts Osteopathic Hospital in 1944. Repeated clinical observations since that time indicate that the linkage more frequently involves the right rib 4 at this spinal level. Findings at T-4 and right rib 4 include immediately increasing local tissue resistance to the following: axial rotation of shoulders and trunk to the left, sidebending to the right, and extension; translations anteriorly, left, and caudally; and inhalation. Opposing asymmetries are present at adjacent (superior and inferior) vertebral and right costal segments 3 and 5 .

In 1977, Larson $^{5}$ carefully described the somatic findings related to cholelithiasis as follows:

In addition to the local changes found by palpating the upper abdomen, somatic changes are usually found in the lower right chest wall and in the lower thoracic spine when gallbladder disease is present. At the tenth thoracic level, the paraspinal tissues will usually display the responses of facilitation of that area to the stimulation of visceral afferent nerves from the gallbladder.

Occasionally, paraspinal responses may be displayed at the ninth or the 11th thoracic segment. The degree of these local signs parallels the acuity or the chronicity of the visceral disorder. They include changes in the skin, subcutaneous fascia, paravertebral musculature and some variation in the mobility at the T-10 segment. It is also common to see and palpate coexisting somatic changes occurring in relation to the tenth rib and its neurologic extension anteriorly into the abdominal wall. In the acute state, there is hyperacuity (response to pin prick or digital pressure) in the area of the skin supplied by the T-10 dermatome. This may include the lateral chest wall and upper abdominal wall. In the immediate area of the T-10 segment, the subcutaneous fascia will display a change in consistency, indicating some change in the circulatory mechanisms. Deeper palpation will indicate a marked increase in muscle tone, most obvious in the deeper, shorter musculature related to the T-10 vertebral segment. This hypertonicity response of the local muscles will effect a change in mobility of the immediate area of the spine.

In patients with cholelithiasis, my clinical testing of the area described has revealed the following characteristics at the T-10 level. Findings of linkage at T-10 with right rib 10 include immediately increasing tissue resistance to the following: axial rotation introduced through the shoulders to the left, sidebending left, and extension; translations posteriorly, left, and caudally; and inhalation. Opposing asymmetries are present at adjacent vertebral and right costal segments 9 and 11 .

For the patient with recurring acute upper gastrointestinal complaint, a frequent somatic component may involve linkage at T- 5 with left rib 5 . Palpable findings include the sense of immediately increasing resistance in response to the following: axial rotation of shoulders to the left, sidebending right, and forward flexion; translations posteriorly, left, and caudally; and exhalation. Magoun ${ }^{6}$ described in detail his approach to osteopathic ma- nipulation of this spinal region in a child with "stomach symptoms of a dyspeptic nature." With the patient in the left sidelying position, he used a final slight direct thrusting force to address primarily the local restriction to sidebending right and translation left. It is interesting to note his careful description of "the proper positioning and relaxation" of the patient in extension, with rotation to the right from above and counter rotation to the left from below-directions of increasing ease.

\section{Discussion}

In the past, observing the response to treatment has provided a valuable indicator of the nature of primary and secondary relationships within the fundamental three-segment unit of dysfunction. Because response to treatment of dysfunctional areas with segmental linkage also has provided insights into viscerosomatic motor changes, this discussion will present comment on (1) features of the treatment procedure and (2) response patterns that convey impressions of acuteness and chronicity. Comments are based mainly on experience with the application of an indirect method in osteopathic manipulative treatment to return asymmetric to symmetric behavior in an area of linkage.

The area of the primary dysfunction with linkage is larger than that of dysfunction without this pattern, because it consists of a vertebral and a costal component acting in response to motion as if the two were linked within a common primary motion defect. For this reason, the physician contacts both primary components at that spinal level: One or two fingers overlie the vertebral transverse process(es) in the central column; another finger (or thumb) contacts the involved rib angle at the same level laterally. Each of the six degrees of freedom, as well as active respiration, are still used in the procedural approach. The goal of treatment continues to be restoration of symmetry, as determined by response to post-treatment motion tests.

After a single osteopathic manipulative procedure, with an effective release and return to a central resting postion, re-examination often reveals much greater change in the primary and secondary elements of the vertebral component than in the costal component. Although retesting of response to motion indicates a general improved mobility, and although palpatory cues of immediate resistance at the primary vertebral segment may now appear very slight, the cues of asymmetry at the three-segment unit of costal dysfunction still may be quite strong and may be more readily elicited in comparison. The reverse of this disproportionate response (that is, the costal component is more reduced and the vertebral cues are more persis- 
tent) is seldom encountered. Repeating and refining the procedure to improve the response will result in a complete return to symmetry throughout both components. Tentatively, this response feature appears to support the conjecture that visceromotor influence on the thoracic tissues may be more focused and intense in its involvement of tissues in the lateral costal column than in the central vertebral column.

Locations of thoracic segmental asymmetry involved in linkage appear to be capable of sufficient response to osteopathic manipulative treatment, so that symmetry is restored when motions are then retested. However, the motion at the linkage site will be asymmetric again on a follow-up visit, but the asymmetry is less intense, more easily responsive to manipulation, and not observable on further follow up. This kind of response, in the case of upper gastrointestinal linkage at T-5, may be associated with a history of recent visceral distress that was locally acute for a short interval but no longer symptomatic, or to a history of mild distress recurring occasionally for a year or longer.

In contrast, the example provided by a 40 -yearold patient with a childhood history of bronchial asthma and a continuing pattern of seasonal allergic airway impairment may be illustrative (the time element is definitive). The T-4 linkage present on initial examination effectively responds to osteopathic manipulative treatment at a given time, but re-examination on follow-up visits invariably reveals the presence of the characteristic asymmetry at T-4. The intensity will be somewhat proportionate to stressful circumstances, whether allergic, physical, or emotional, during the interval between examinations; the detailed motion characteristics of the asymmetry, however, will remain unchanged.

Repeated response to specific osteopathic manipulative treatment, as part of a management program, contributes to clinical progress in (1) reduction of musculoskeletal complaint in the neck and upper part of the spine, (2) greater range of respiratory ease, (3) decrease in degree and frequency of remissions, and (4) reduction in the need for medicinal support. Still, the characteristic behavior at T- 4 can be predicted on periodic re-examination; only the intensity varies.

These examples of variability in the response to osteopathic manipulative treatment suggest the need for a rationale regarding acuteness and chronicity of this reflex motor manifestation of visceral afferent input. Once established at a spinal vertebral level as a local restricted function in response to motion tests, that segment's conflict with every- day movement patterns becomes obvious: It complies with some directions but resists most. My view is that this conflict may lead to a continuing acute disturbance in the condition of the somatic tissues at the asymmetric segment. The evidence for this concept becomes apparent on examination of the segmental soft tissues. The palpatory impressions of acuteness in somatic tissues have been frequently described as increased muscular fullness or ropiness, increased subcutaneous fluid or bogginess, increased skin surface friction or drag, and increased skin warmth. Each tissue characteristic reflects elements of an acute congestive phase.

When the segment's conflict with body movements continues in time, as in the linkage at the T-4 level in the 40-year-old asthmatic patient, the asymmetric structural mobility becomes more restricted. It is associated with deep, local muscular tension and decreased fullness (mass), sensed as an increased resistance and decreased elasticity to palpation with deep pressure. The local subcutaneous tissue is sparse, is represented often by local skin surface depression (valley), and accompanied by increased connective tissue resistance to shearing of the superficial skin surface over the underlying muscle. Although the motor conflict continues acutely during movement, the deeper tissue changes in time become chronic, and range of motion becomes more limited. The well-established nature of the process is reflected in the stability of the asymmetric characteristics when response to an extended period of osteopathic manipulative management is monitored.

Providing an effective rationale for the predictable stability and complexity of the motion asymmetries presented in chronic linkage is not simple. In the chronic asthmatic patient, for example, the seven characteristics of individual motion asymmetry at T-4 remain unchanged, in sprite of alocal short-term responses toward symmetry and reduced intensity after osteopathic manipulative treatment. No anatomic concept of fascial, ligamentous, muscular, or joint tissue defect alone would seem to account for the precise nature of the asymmetric behavior.

What element of neuromusculoskeletal control can dictate such a specific asymmetric behavior? It is as if there were capability within the mobile system to program each detail of a segment's asymmetric behavior, and then to maintain the individuality of that response pattern, apparently without relief. The point of neural control appears to be segmental, at the spinal cord level. The altered control involves thresholds at those synapses where regulatory (inhibition/excitation) changes influence 
the transmission of impulses that normally account for the compliance of mobile segments during body movements.

\section{Summary}

Findings of somatic dysfunction associated with somatic and visceral inputs have been differentiatied based on tests for motor asymmetries at dysfunctional segments. In these palpatory studies of the somatic system, tests for segmental definition follow on the initial screening tests of physical examination - at the level of the differential diagnosis in medical problem solving. The location of segmental dysfunctions, and the characteristics of their motor asymmetries have provided criteria for assessment of findings. For the physician, these aspects are central to the clinical investigation and clinical management of somatic dysfunction.
1. Johnston WL, Kelso AF, Hollandsworth DL, et al: Somatic manifestations in renal disease: A clinical research study. JAOA 1987;87:22-35. 2. Johnston WL: Segmental behavior during motion: III. Extending behavioral boundaries. JAOA 1973;72:462-474

3. Johnston WL, Hill JL, Sealey JW, et al: Palpatory findings in the cervicothoracic region: Variations in normotensive and hypertensive subjects. A preliminary report. JAOA 1980;79:300-308.

4. Johnston WL, Hill JL, Elkiss ML, et al: Identification of stable somatic findings in hypertensive subjects by trained examiners using palpatory examination. JAOA 1982;81:830-836.

5. Larson NJ: Manipulative care before and after surgery. Osteopathic Med, January 1977, pp 41-49.

6. Magoun HI: Gastroduodenal ulcers from the osteopathic viewpoint. $A A O$ Year Book, 1962, pp 117-120

Dr. Johnston is professor, Department of Family Medicine, Michigan State University - College of Osteopathic Medicine, E Lansing Mich.

Dr. Johnston, MSU-COM, W Fee Hall, E Lansing, Mich 48824. 


\section{The National Osteopathic Foundation Announces:}

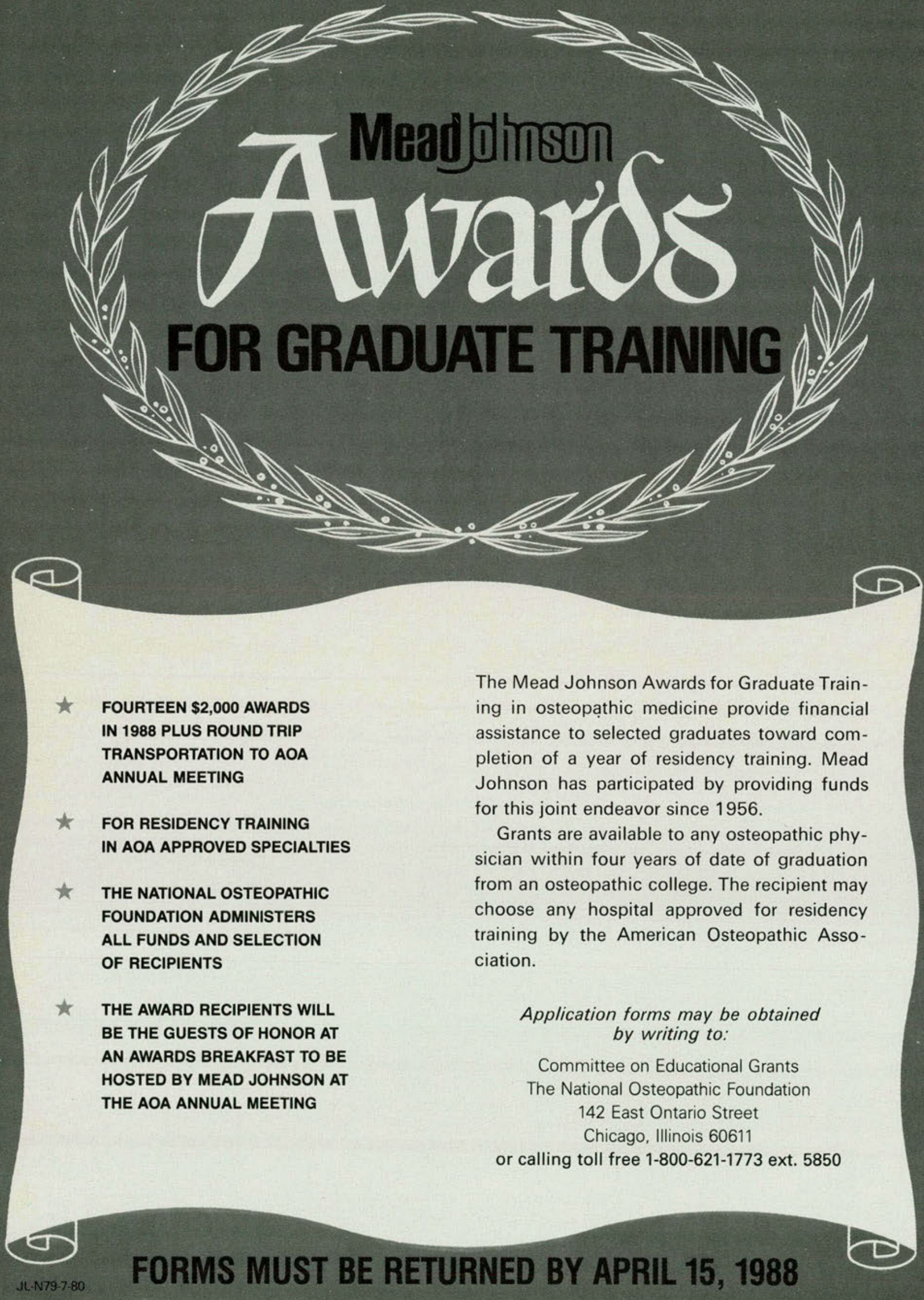




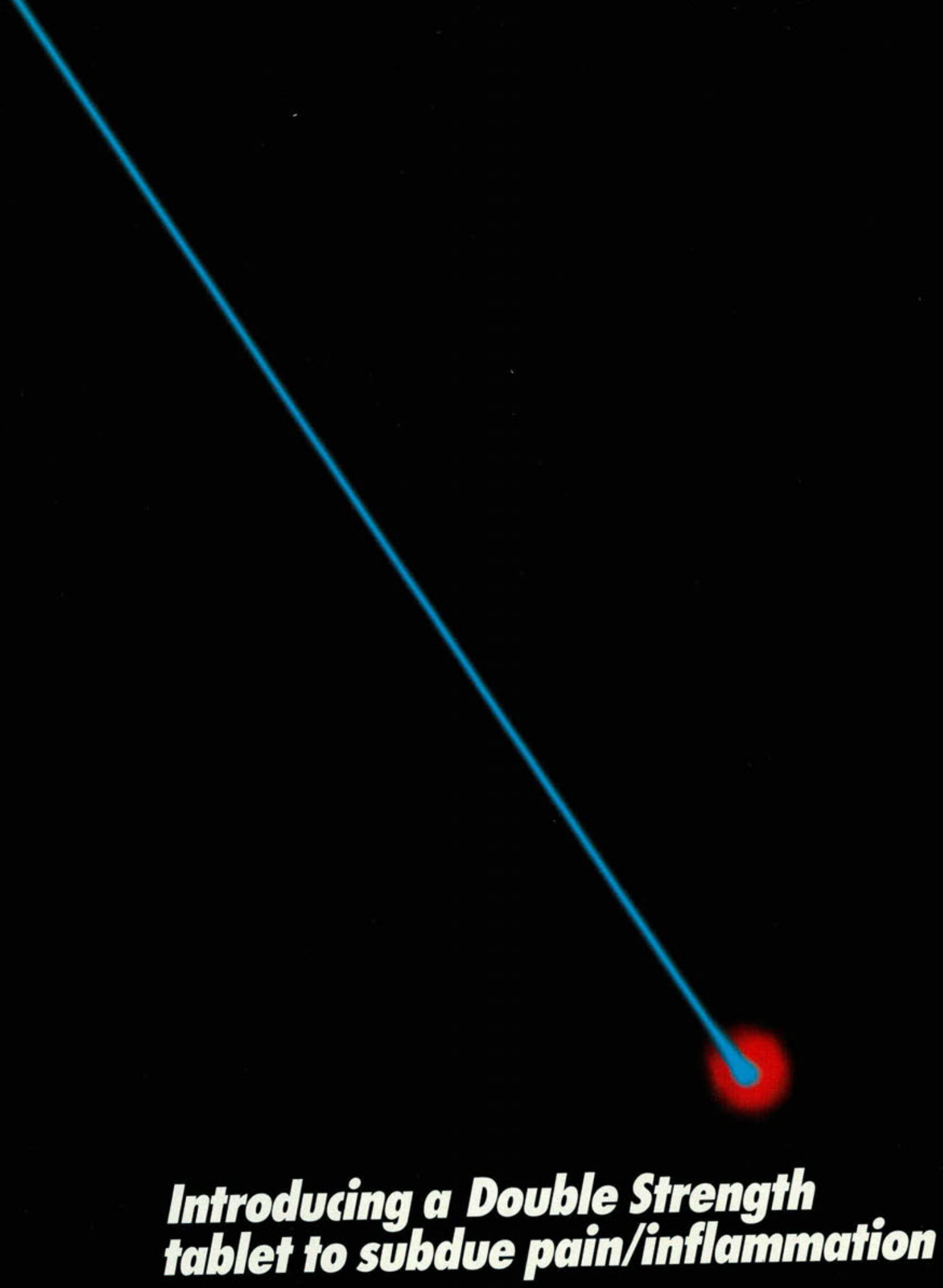

\section{Introducing a Double Strength tablet to subdue pain/inflammation}




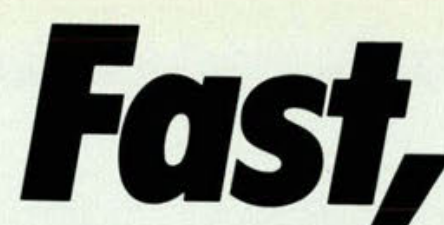

poin/inilat

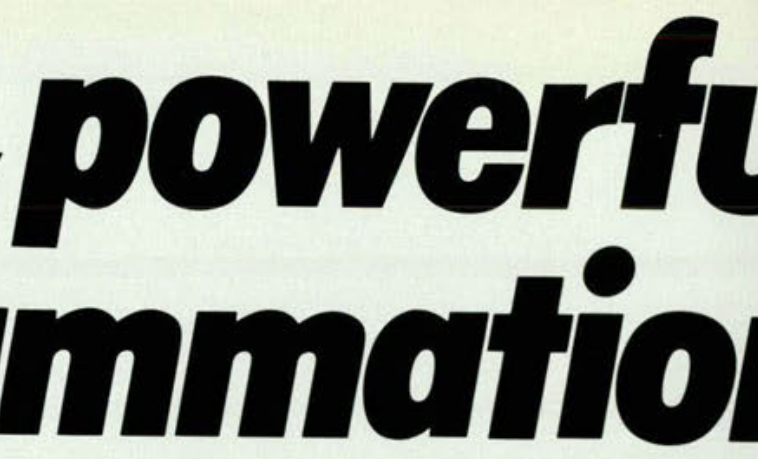

Now, there is ANAPROX Double Strength that gives you more analgesic/anti-inflammatory activity per dose. It joins the proven ANAPROX $275 \mathrm{mg}$ strength to provide you with greater dosage flexibility and convenience.

- Twice the strength-550 mg ANAPROX per tablet.

- Dosage- The daily dosage of $1650 \mathrm{mg}$ can be used for limited periods when a higher level of analgesic/anti-inflammatory activity is required. Do not exceed this daily dose.

- Greater analgesic/anti-inflammatory activity. in each tablef-reduces inflammation as it relieves the pain.

- No oral analgesic works faster-onset of pain relief may occur as fast as 20 minutes.

- Efficacy equal to the narcotic combinationseffective analgesia with a potent anti-inflammatory bonus the codeine combinations and propoxyphene napsylate can't offer.

- Non-narcotic-no narcotic-type side effects* or clinical limitations.

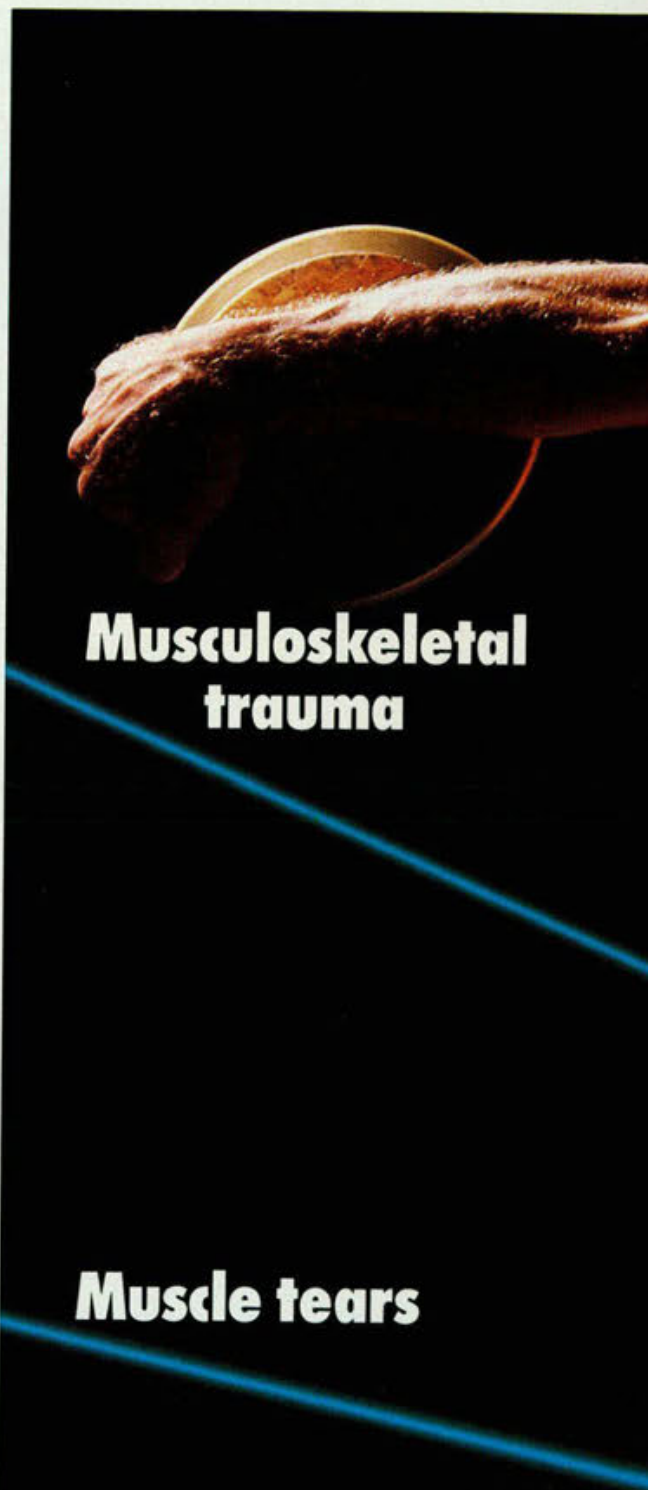

Sprains \& strains 
efer forthe
ov treat every doy
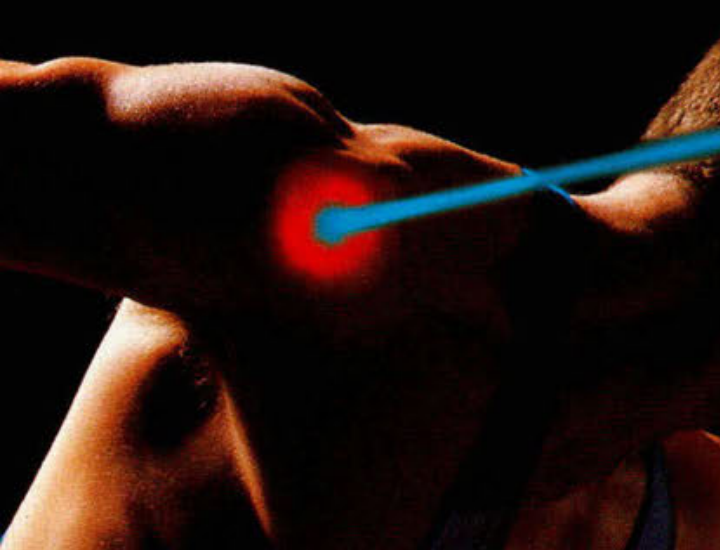

\section{Bursitis}

\section{Tendinitis}

\section{Joint injury}

Iniroduding 


\section{Fast, powerful relief for the pain inflammation you see every day}

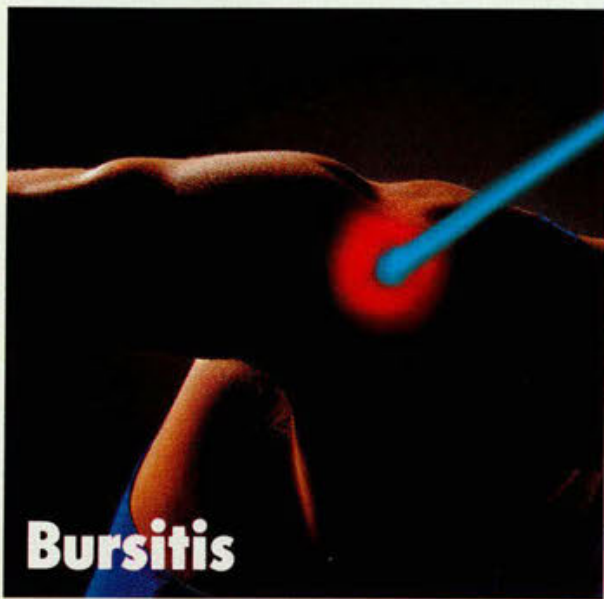

\section{Musculoskeletal trauma}

ANAPROX ${ }^{\circ}$ and ANAPROX $\odot$ DS (naproxen sodium) Tablets

Brief Summary

Indications: Relief of mild to moderate pain; treatment of primary dysmenorrhea. Treatment of rheumatoid arthritis, osteoarthritis, juvenile arthritis, ankylosing spondylitis, tendinitis and bursitis, and acute gout. Contraindications: Patients who have had allergic reactions to NAPROSWN. ANAPROX or ANAPROX DS or in whom aspirin or other NSAIDs induce the syndrome of asthma, rhinitis, and nasal polyps. Because anaphylactic reactions usually occur in patients with a history of such reactions, question patients for usually occur in patients with a history of such reactions, question patients for
asthma, nasal polyps, urticaria, and hypotension associated with NSAIDS asthma, nasal polyps, urticaria, and hypotension associated with $\mathrm{N}$.
before starting therapy. If such symptoms occur, discontinue the drug. Warnings: Peptic ulcers and GI bleeding have been reported in patients on NSAIDs, including naproxen sodium. In patients with GI bleeding or active petic ulcer, start an anti-ulcer regimen, weigh benefitts and risks of treatment, an monitor patient. Give to patients with history of Gl disease only under close supervision and after reading Adverse Reactions section.

Precautions: DO NOT GIVE NAPROSYN" (NAPROXEN) CONCOMITANTIY WTTH AMAPROX`OR AMAPROX` DS (NAPROXEN SODIUM) SINCE BOTH CIRCULATE IN PLASMAAS THE NAPROXEN ANION. Acute interstitial nephritis with hematt ria, proteinuria, and nephrotic syndrome has been reported. Patients with impaired renal function, heart faliure, liver dysfunction, taking diuretics, an the elderly are at greater risk of overt renal decompensation. If this occurs, dis continue the drug. Use with caution and monitor serum creatinine and/or creal: nine clearance in patients with significantly impaired renal function. Use caution in patients with baseline creatinine clearance less than $20 \mathrm{~m} /$ minut Use the lowest effective dose in the elderly or in patients with chronic alcoholic liver disease or cirrhosis. With NSAIDs, borderline elevations of fiver tests may occur in up to $15 \%$ of patients. They may progress, remain unchanged, or be transient with continued therapy. Elevations of SGPT or SGOT occurred in con trolled clinical trials in less than $1 \%$ of patients. Severe hepatic reactions including jaundice and fatal hepatitis, have been reported rarely. If liver disease develops or if systemic manifestations occur (e. g, easinophilia or rash), discon. tinue therapy. If steroid dosage is reduced or eliminated during therapy, do so slowly and observe patients closely for adverse effects, including adrenal insuficiency and exacerbation of arthritis symptoms. Determine hemoglobin values periodically for patients with initial values of 10 grams or less who receive long. term therapy. Peripheral edema has been reported. For patients with restricted
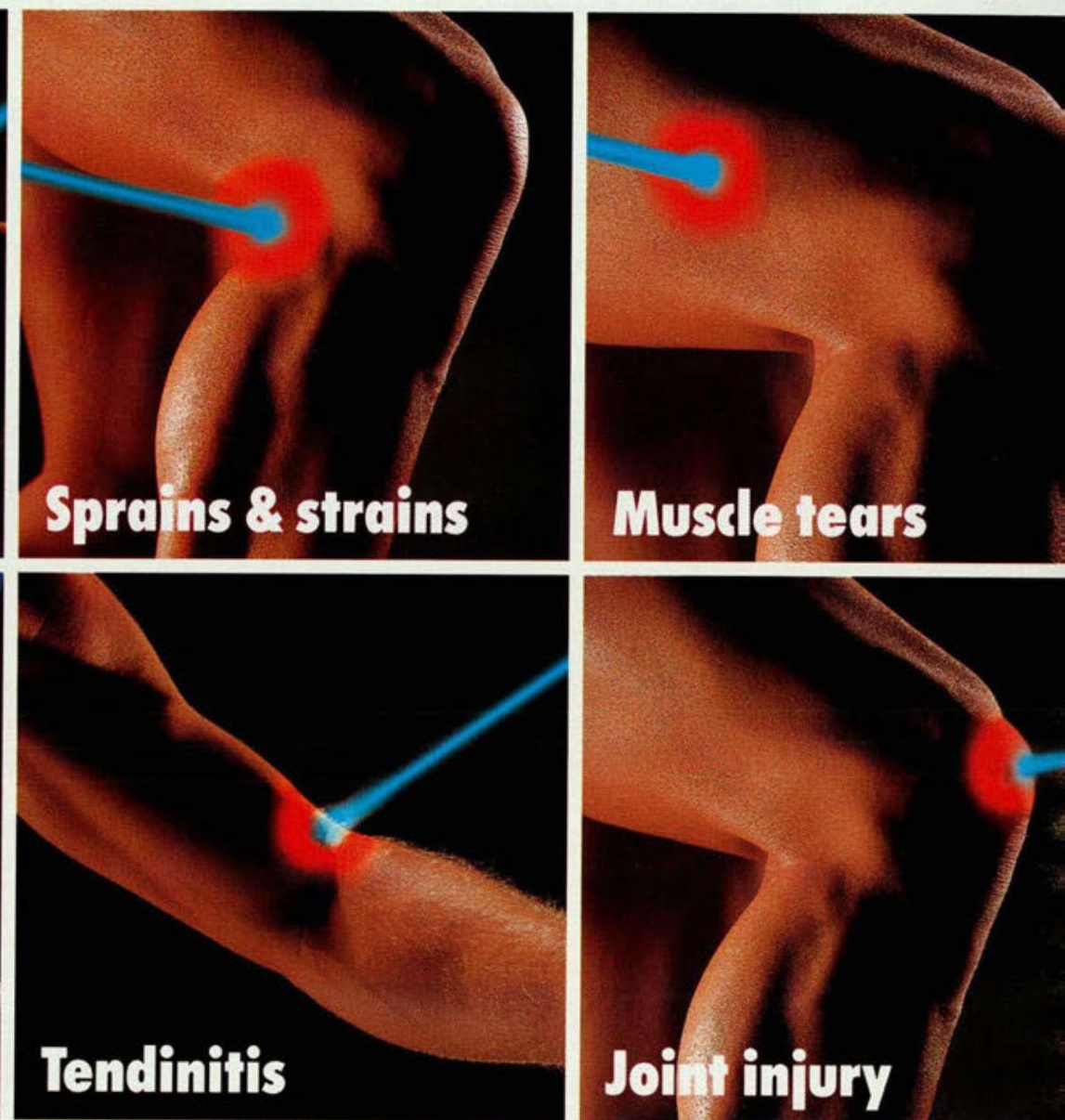

sodium intake, note that each tablet contains approximately $25 \mathrm{mg}$ or $50 \mathrm{mg}$ (1 or $2 \mathrm{mEq}$ ) sodium. Use with caution in patients with fluid retention, hyperten sion or heart failure. The drug's antipyretic and anti-inflammatory activities may reduce fever and inflammation, diminishing their diagnostic value. Con duct ophthalmic studies if any change or disturbance in vision occurs. Information for Patients: Patients should use caution for activities requiring alertness if they experience drowsiness, dizziness, vertigo or depression during therapy

Drug Interactions: Use caution when giving concomitantly with coumarinOrug Interactions: Use caution when giving concomitantly with coumarin. type anticoagulants; a hydantoin, sulfonamide or s

ium; beta-blockers; probenecid; or methotrexate. gation and prolong bleeding time or increase urinary values for 17 -ketogenic steroids. Temporarily stop therapy for 72 hours before doing adrenal function tests. The drug may interfere with urinary assays of 5 HIAA.

Carcinogenesis: A 2-year rat study showed no evidence of carcinogenicity Pregnancy: Category B. Do not use during pregnancy unless clearly needed. Avoid use during late pregnancy.

Nursing Mothers: Avoid use in nursing mothers.

Pediatric Use: Single doses of $25-5 \mathrm{mg} / \mathrm{kg}$ (as naprowen suspension), with total daily dose not exceeding $15 \mathrm{mg} / \mathrm{kg}$ /day. are safe in children over 2 years total daily

of age

Adverse Reactions: In a study. Gl reactions were more frequent and severe in rheumatoid arthritis patients on $1500 \mathrm{mg} / \mathrm{day}$ naproxen than in those on 750 $\mathrm{mg} /$ day (doses comparable to $1650 \mathrm{mg}$ and $825 \mathrm{mg}$ of naproxen sodium). In studies in children with juvenile arthritis, rash and prolonged bleeding times were more frequent, Gl and CNS reactions about the same, and other reactions less frequent than in adults. Incidence Greater Than 1\%, Probable Causal Relationship: Gl: The most frequent complaints related to the GI tract: constipa. tion* heartburn*" abdominal pain * nausea* dyspepsia diarrhea stomatitis. CNS: headache," diziness," drowsiness", light-headedness, vertigo. Derma. tologic: itching (pruritus)" skin eruptions," ecchymoses, sweating, purpura.
Special Senses: tinnitus," hearing disturbances, visual disturbances. CardioSpecial Senses: tinnitus;" hearing disturbances, visual disturbances. Cardio-
vascular: edema, dyspnea," palpitations, General: thirst. "Incidence of vascular: edema*, dyspnea, palpitations. General: thirst. "Incidence of reported reaction $3 \%-9 \%$. Where unmarked, incidence less than $3 \%$. Incidence Less Than 1\%: Probable Causal Relationship: Gl: abnormal liver function tests, colitis, GI bleeding and/or perforation, hematemesis, jaundice, melena, peptic ulceration with bleeding and/or perforation, vomiting Renal: glomerular

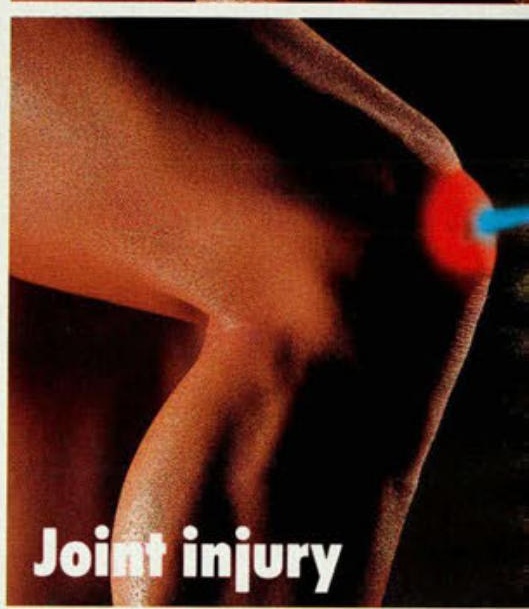

nephritis, hematuria, interstitial nephritis, nephrotic syndrome, renal disea Hematologic: agranulocytosis, eosinophilia, granulocytopenia, leukopeni thrombocytopenia. CNS: depression, dream abnormalities, inability to conce trate, insomnia, malaise, myalgia and muscle weakness. Dermatologic: alop cia, photosensitive dermatitis, skin rashes. Special Senses: hearin impairment. Cardiovascular: congestive heart failure. Respiratory: eosinophit pneumonitis. General: anaphylactoid reactions, menstrual disorders, pyrex (chills and fever). Causal Relationship Unknown: Hematologic: aplastic anemi hemolytic anemia. CNS: cognitive dysfunction. Dermatologic: epidermal necr lysis, enythema multiforme, Stevens-Johnson syndrome, urticaria. Gl: ulcerativ stomatitis. Cardiovascular: vasculitis. General: angioneurotic edema, hyp glycemia, hypoglycemia

Overdosage: May have drowsiness, heartburn, indigestion, nausea, vomitin Empty stomach and use usual supportive measures. Prompt administration 5 grams activated charcoal may reduce drug absorption. Hemodialysis may b appropriate in renal failure.

Dosage and Administration for Mild to Moderate Pain, Dysmenorrhea an Acute Tendinitis and Bursitis: The recommended starting dose is $550 \mathrm{mg}$. fo lowed by $275 \mathrm{mg}$ every 6 to 8 hours, as required. The total daily dose should no exceed $1375 \mathrm{mg}$.

Dosage and Administration for Rheumatoid Arthritis, Osteoarthritis an Ankylosing Spondylitis: The recommended dose in adults is $275 \mathrm{mg}$ or $550 \mathrm{~m}$ twice daily. In patients who tolerate lower doses well, the dose may be increase to $1,650 \mathrm{mg}$ per day for limited periods when a higher level of an inflammatory/analgesic activity is required. At this dosage, physicians shoul observe sufficient increased clinical benefits to offset potential increased ris Caution: Federal law prohibits dispensing without prescription. See package insert for full Prescribing Information. (C) Revised 10:8 S SYNTEK

\section{Anaprox'D5} (NAPROXEN SODIUM) 